\title{
PERFORMANCE EVALUATION OF A SINGLE-MODE BIOMETRIC ACCESS CONTROL SYSTEM
}

\author{
${ }^{1}$ Bopatriciat Boluma Mangata, ${ }^{2}$ Mbuyi Mukendi Eugène, ${ }^{3}$ Batubenga Mwamba Nzambi, ${ }^{4}$ Crispin \\ Bukanga Maheshe, ${ }^{5}$ Parfum Bukanga Christian \\ ${ }^{I}$ Teaching Assistant, Department of Mathematics and Computer Science, University of Kinshasa, Kinshasa, DR Congo \\ ${ }^{2}$ PhD, Department of Mathematics and Computer Science, University of Kinshasa, Kinshasa, DR Congo \\ ${ }^{3}$ PhD, Department of Mathematics and Computer Science, University of Kinshasa, Kinshasa, DR Congo \\ ${ }^{4}$ Teaching Assistant, Department of Mathematics and Computer Science, University of Kinshasa, Kinshasa, DR Congo \\ ${ }^{5}$ Teaching Assistant, Department of Mathematics and Computer Science, University of Kinshasa, Kinshasa, DR Congo \\ Email: $\left\{^{1}\right.$ bopatriciat.boluma@unikin.ac.cd, ${ }^{2}$ mbuyieugene@gmail.com, ${ }^{3}$ jude.batubenga@unikin.ac.cd, \\ ${ }^{4}$ crispinbukanga@gmail.com, ${ }^{5}$ parfum.bukanga@unikin.ac.cd\}
}

\begin{abstract}
The present work evaluates the performance of a fingerprint-based access control system to secure premises. In order to evaluate these performances, we used a regression model in which the dependent variable is of qualitative type, more precisely, the variable: "the new individual who takes the entrance test is positive or negative".Our model is therefore a classifier capable of diagnosing whether fingerprints will be accepted or not. This performance evaluation model is realized by means of the confusion matrix, the calculations of the evaluation parameters (Sensitivity, Specificity, Positive Predictive Value, Negative Predictive Value and False Negative), and finally the plots of the sensitivity values against 1-Specificity (ROC curve).On a sample of six hundred individuals of which 470 enrolled and 130 not enrolled, the access control system obtained the results of which 456 true positives, 14 false negatives, 10 false positives and 120 true negatives which constitute our confusion matrix, which we were able to evaluate the performance of our system by applying the calculations of the evaluation parameters.
\end{abstract}

Keywords: System performance, Access Control, Fingerprinting, Sensitivity, Specificity, Positive Predictive Value, Negative Predictive Value, False Negative, ROC curve, Confusion Matrix.

\section{Introduction}

\subsection{Problematic}

When assessing the diagnostic value of a sign for a condition, the sign is sought in individuals with and without the condition in question [6].

Two experimental situations are to be considered:

$\checkmark$ A representative sample of a population is taken. From this sample, we can estimate all the probabilities of events by the corresponding observed frequencies;

$\checkmark$ Two samples are taken, one representative of the individuals for whom the diagnosis is true, the other representative of the individuals for whom it is false. This is often the only way to proceed in practice, especially when the disease under consideration is rare. It should be noted, however, that it is no longer possible to estimate any probability by the corresponding observed frequency $[6,15]$.

The real problems that arise in controlling access to students in a multi-entry institution, especially when verifying academic fee payment slips, must be assessed to determine the acceptability or otherwise of the system.

Thus, in order to motivate the continuation of this work, questions of the kind listed below will not be excluded:
What are the most effective methods we can apply to properly evaluate the performance of the access control system?

$\checkmark$ How can the problem of access control performance to secure premises by fingerprints be properly assessed?

These questions constitute the set of problems that we will attempt to examine in the following lines.

\subsection{Assumptions}

To solve these problems, the optimal solution that we propose in this work is to evaluate the performance of this system, using a regression model where the dependent variable is of qualitative type, more precisely, it is the variable: "the new individual who makes the entry test is positive or negative".

Specifically, we will:

$\Leftrightarrow$ To design a model that would be a classifier that could diagnose whether the impression is accepted or not.

(4) To make a confusion matrix, to calculate the parameters of the evaluation (Sensitivity, Specificity, the positive predictive value, the negative predictive value and the false negative), and finally to plot the values of the sensitivity Se as a function of 1-Sp (ROC curve), which will have the purpose of well evaluating the performance of our system. 
1.3. Lenses

\subsubsection{General objective}

The overall objective of this work is to design a tool that will allow the evaluation of the access control management system.

\subsubsection{Specific objectives}

Our approach will achieve the following objectives:

* Implement a classifier that can diagnose whether the fingerprint is accepted or not;

* Design a system performance evaluation model based on the confusion matrix, calculations of the evaluation parameters (Sensitivity, Specificity, Positive Predictive Value, Negative Predictive Value and False Negative), and finally the plots of the sensitivity values Se versus 1-Sp (ROC curve)

\subsection{Interest of the subject}

The interest of such an approach is to make a major contribution to the scientific community, by providing them with a logical approach to evaluate the performance of a fingerprint-based access control system to secure premises.

\section{Presentation of the problem}

Let $A$ (Set of individuals enrolled in the access control system) and $B$ (Set of individuals not enrolled in the access control system) be any two events of a fundamental set $E$ consisting of six hundred individuals, with a probability distribution IP [1, 2].

We are interested in what happens to the probability of $A$ when we learn that $B$ has already been realized and the probability of $B$ when we learn that $A$ has already been realized, i.e. when we restrict the set of possible outcomes $E$ to $B$ and $E$ to $A[\mathbf{1 0}, \mathbf{1 6}]$.

The conditional probability of $A$, knowing that event $B$ is realized, is denoted $\operatorname{IP}(A / B)$ and is defined by the following relation:

$$
\operatorname{IP}(\mathrm{A} / \mathrm{B})=\frac{\mathrm{IP}(\mathrm{A} \cap \mathrm{B})}{I P(B)}
$$

The conditional probability of $B$, knowing that event $A$ is realized, is noted $\operatorname{IP}(B / A)$ and is defined by the following relation:

$$
\operatorname{IP}(\mathrm{B} / \mathrm{A})=\frac{\mathrm{IP}(\mathrm{B} \cap \mathrm{A})}{I P(A)}
$$

In these equations, the probabilities of the events $A \cap B, \mathrm{~A}$ and $\mathrm{B}$ must be calculated over the entire fundamental set $E$.

Consider E, the experiment that consists in verifying the performance of an access control system on a fundamental set $\mathrm{E}$ of six hundred students of the University of Kinshasa

ISSN (Print): 2456-6411 | ISSN (Online): 2456-6403
[3].

The probabilities of True Positive, False Positive, False Negative and True Negative events have certain values.

The Venn diagram below shows the distribution of each event:

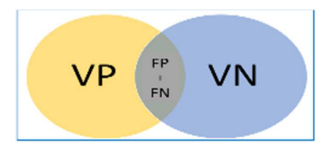

Figure 2-1: The Venn diagram of the set $\mathrm{E}$.

\subsection{Estimation of evaluation parameters}

\subsubsection{A representative sample}

\subsubsection{The data}

When we have a representative sample of a population, we can summarize the data of the experiment by a $2 \times 2$ confusion matrix, on which the numbers are indicated.

Given two fundamental subspaces of the space E, the random events are $\mathrm{B}_{1}$ : "being enrolled" and $\mathrm{B}_{2}$ : "not being enrolled".

Let VP, FP, FN and VN be the events from the fundamental set E.

The elements of E, possible results of the tests are as follows $[7,8]$ :

$\checkmark$ VP (True Positives), represents the individuals enrolled $(E+)$ and accepted by the system $\{S\}$;

$\checkmark$ FP (False Positives), represents the individuals not enrolled (E-) and in whom the system has accepted them $\{\mathrm{S}\}$;

$\checkmark$ FN (False Negatives), represents the individuals enrolled $(E+)$ and in whom the system has rejected them $\{\mathrm{S}-\}$;

$\checkmark$ VN (True Negatives), represents individuals who were not enrolled $(E-)$ and where the system rejected them $\{\mathrm{S}-\}$;

\subsubsection{Confusion matrix}

In supervised machine learning, the confusion matrix is a matrix that measures the quality of a classification system. Each row corresponds to a real class, each column corresponds to an estimated class. The cell row L, column C contains the number of elements of the real class $L$ that have been estimated as belonging to the class $C[\mathbf{1 1}, \mathbf{1 3}]$.

One of the interests of the confusion matrix is that it quickly shows whether a classification system manages to classify correctly.

Table 1: Confusion Matrix

\begin{tabular}{|l|c|c|}
\hline & E+ & E- \\
\hline 272 & JREAS, Vol. 07, Issue 01, Jan 22
\end{tabular}




\begin{tabular}{|c|c|c|}
\hline $\mathrm{S}+$ & VP & FP \\
\hline $\mathrm{S}-$ & FN & VN \\
\hline
\end{tabular}

With that, our sample is as follows:

Table 2: Sample Confusion Matrix

\begin{tabular}{|c|c|c|}
\hline & E+ & E- \\
\hline S+ & 456 & 10 \\
\hline S- & 14 & 120 \\
\hline
\end{tabular}

\subsubsection{Estimation of sensitivity and specificity}

The sensitivity of a sign for a disease is the probability that the sign will be present if the subject has the disease in question. It is therefore the conditional probability that can be noted [14]:

By definition, the Sensitivity $=\mathrm{Se}=\mathrm{IP}(\mathrm{S} / E)$

This conditional probability is estimated by the ratio of the corresponding numbers to the observed confusion matrix:

$$
S e \approx \frac{V P}{V P+F N}
$$

Note: True parameters, which are conditional probabilities, and their estimates, which are ratios of observed numbers, are noted identically, following established usage [5].

The specificity of an access control test is the probability that the individual will not be enrolled and the system will deny access.

$$
\text { Specificity }=\operatorname{Sp}=\operatorname{IP}(\bar{S} / \bar{E}) \approx \frac{V N}{V N+F P}
$$

A diagnostic test is therefore all the more specific as subjects free of the disease present the $\mathrm{S}$ sign less often.

For a "perfect" test, i.e. one that makes no errors, the sensitivity and specificity values are equal to 1 .

\subsubsection{Estimation of predictive values}

In practice, let's assume, when a doctor receives the result of a complementary examination, positive or negative, he does not know whether the patient suffers from the condition he is trying to diagnose or not, and the probabilities he is interested in are expressed as follows: what is the probability of the presence of disease $\mathrm{E}$ in this patient, knowing that the examination has given a positive (or negative) result?. These probabilities are called predictive values $[2,14]$.

Specifically, we have:

$\checkmark \quad$ The positive predictive value (VPP) of a sign for a disease is the probability that the subject will have the disease if the sign is present;

ISSN (Print): 2456-6411 | ISSN (Online): 2456-6403
The negative predictive value (VPN) of a sign for a disease is the probability that the subject is free of the disease if the sign is absent [14].

The estimates are obtained from the same data table:

$$
\begin{aligned}
& V P P=\operatorname{IP}(\mathrm{E} / \mathrm{S}) \approx \frac{\mathrm{VP}}{V P+F P} \\
& V P N=\operatorname{IP}(\bar{E} / \bar{S}) \approx \frac{V N}{V N+F N}
\end{aligned}
$$

\subsubsection{Calculation of the evaluation parameters}

2.2.1.5.1. Calculation of the parameters of the evaluation on the fundamental set $E$

Let us compute the estimators of these parameters in the case where we want to evaluate the performance of the access control system on the fundamental set $\mathrm{E}$, consisting of six hundred individuals distributed in the confusion matrix below:

Table 3: Individuals distributed in the Confusion Matrix

\begin{tabular}{|c|c|c|}
\hline & E+ & E- \\
\hline S+ & 456 & 10 \\
\hline S- & 14 & 120 \\
\hline
\end{tabular}

$$
\begin{aligned}
& S e=\frac{\mathrm{VP}}{\mathrm{VP}+\mathrm{FN}} \Rightarrow S e=\frac{456}{(456+14)} \Rightarrow S e=0.97021277 \\
& S p=\frac{\mathrm{VN}}{\mathrm{VN}+\mathrm{FP}} \Rightarrow S p=\frac{120}{(120+10)} \Rightarrow S p=0.92307692 \\
& V P P=\frac{\mathrm{VP}}{\mathrm{VP}+\mathrm{FP}} \Rightarrow V P P=\frac{456}{(456+10)} \Rightarrow V P P=0.97854077 \\
& V P N=\frac{\mathrm{VN}}{\mathrm{VN}+\mathrm{FN}} \Rightarrow V P N=\frac{120}{(120+14)} \Rightarrow V P P=0.89552239 \\
& \Rightarrow F N=1-\mathrm{SP} \\
& \Rightarrow F N=1-0.92307692 \\
& \Rightarrow F N=0.07692308
\end{aligned}
$$

\subsection{Calculation of the evaluation parameters on the partitions of $E$}

Consider events $A_{1} \ldots A_{n}$ such that they form a partition of the fundamental set $E$ [7]. By definition, $A_{i}$ are mutually exclusive and their union is $E$ :

$$
\begin{gathered}
\forall(i \neq j),\left(A_{i} \cap A_{j}=\varnothing\right) ; \\
\bigcup_{j=1}^{n} A_{i}=E
\end{gathered}
$$

The following table illustrates the composition of our fifteen events that create the partition of the fundamental set $\mathrm{E}$ :

Table 4: Fifteen events create the partition of the fundamental set $\mathrm{E}$. 


\begin{tabular}{|c|c|c|c|c|c|}
\hline Num & VP & FN & FP & VN & Total \\
\hline 1 & 36 & 0 & 1 & 5 & 42 \\
\hline 2 & 45 & 1 & 1 & 7 & 54 \\
\hline 3 & 36 & 1 & 1 & 7 & 45 \\
\hline 4 & 35 & 1 & 1 & 7 & 44 \\
\hline 5 & 33 & 1 & 1 & 8 & 43 \\
\hline 6 & 32 & 1 & 1 & 8 & 42 \\
\hline 7 & 31 & 1 & 1 & 9 & 42 \\
\hline 8 & 30 & 1 & 1 & 9 & 41 \\
\hline 9 & 29 & 1 & 1 & 10 & 41 \\
\hline 10 & 28 & 1 & 1 & 12 & 42 \\
\hline 11 & 27 & 1 & 0 & 7 & 35 \\
\hline 12 & 25 & 1 & 0 & 5 & 31 \\
\hline 13 & 24 & 1 & 0 & 6 & 31 \\
\hline 14 & 23 & 1 & 0 & 7 & 31 \\
\hline 15 & 22 & 1 & 0 & 13 & 36 \\
\hline Total & 456 & 14 & 10 & 120 & 600 \\
\hline
\end{tabular}

Let us compute the estimators of these parameters in the case where we seek to evaluate the performance of the access control system.

For given thresholds $S_{1}, S_{2} \ldots S_{15}$, and Average (AVG), we obtain the table below $(\mathrm{Se}=\mathrm{VP} /(\mathrm{VP}+\mathrm{FN}), \quad \mathrm{Sp}=\mathrm{VN} /(\mathrm{VN}+\mathrm{FP})$, $\mathrm{VPP}=\mathrm{VP} /(\mathrm{VP}+\mathrm{FP}), \mathrm{VPN}=\mathrm{VN} /(\mathrm{VN}+\mathrm{FN})$, and $\mathrm{FN}=1-\mathrm{Sp})$ :

Table 5: Calculation of estimating parameters.

\begin{tabular}{|c|c|c|c|c|c|}
\hline Group & $\mathrm{Se}$ & $\mathrm{Sp}$ & VPP & VPN & FN \\
\hline$S_{1}$ & 1 & $\begin{array}{l}0,833333 \\
33\end{array}$ & $\begin{array}{l}0,972972 \\
97\end{array}$ & 1 & $\begin{array}{l}0,166666 \\
67\end{array}$ \\
\hline$S_{2}$ & $\begin{array}{l}0,978260 \\
87\end{array}$ & 0,875 & $\begin{array}{l}0,978260 \\
87\end{array}$ & 0,875 & 0,125 \\
\hline $\mathrm{S}_{3}$ & $\begin{array}{l}0,972972 \\
97\end{array}$ & 0,875 & $\begin{array}{l}0,972972 \\
97\end{array}$ & 0,875 & 0,125 \\
\hline $\mathrm{S}_{4}$ & $\begin{array}{l}0,972222 \\
22\end{array}$ & 0,875 & $\begin{array}{l}0,972222 \\
22\end{array}$ & 0,875 & 0,125 \\
\hline$S_{5}$ & $\begin{array}{l}0,970588 \\
24\end{array}$ & $\begin{array}{l}0,888888 \\
89\end{array}$ & $\begin{array}{l}0,970588 \\
24\end{array}$ & $\begin{array}{l}0,888888 \\
89\end{array}$ & $\begin{array}{l}0,111111 \\
11\end{array}$ \\
\hline$S_{6}$ & $\begin{array}{l}0,969696 \\
97\end{array}$ & $\begin{array}{l}0,888888 \\
89 \\
99\end{array}$ & $\begin{array}{l}0,969696 \\
97 \\
9\end{array}$ & $\begin{array}{l}0,888888 \\
89\end{array}$ & $\begin{array}{l}0,111111 \\
11\end{array}$ \\
\hline$S_{7}$ & 0,96875 & 0,9 & 0,96875 & 0,9 & 0,1 \\
\hline$S_{8}$ & $\begin{array}{l}0,967741 \\
94\end{array}$ & 0,9 & $\begin{array}{l}0,967741 \\
94\end{array}$ & 0,9 & 0,1 \\
\hline $\mathrm{S}_{9}$ & $\begin{array}{l}0,966666 \\
67\end{array}$ & $\begin{array}{l}0,909090 \\
91\end{array}$ & $\begin{array}{l}0,966666 \\
67\end{array}$ & $\begin{array}{l}0,909090 \\
91\end{array}$ & $\begin{array}{l}\begin{array}{l}0,090909 \\
09\end{array} \\
\end{array}$ \\
\hline$S_{10}$ & $\begin{array}{l}0,965517 \\
24\end{array}$ & $\begin{array}{l}0,923076 \\
92\end{array}$ & $\begin{array}{l}0,965517 \\
24\end{array}$ & $\begin{array}{l}0,923076 \\
92\end{array}$ & $\begin{array}{l}0,076923 \\
08\end{array}$ \\
\hline$S_{11}$ & $\begin{array}{l}0,964285 \\
71\end{array}$ & 1 & 1 & 0,875 & 0 \\
\hline$S_{12}$ & $\begin{array}{l}0,961538 \\
46\end{array}$ & 1 & 1 & $\begin{array}{l}0,833333 \\
33\end{array}$ & 0 \\
\hline$S_{13}$ & 0,96 & 1 & 1 & $\begin{array}{l}0,857142 \\
86\end{array}$ & 0 \\
\hline $\mathrm{S}_{14}$ & 0,958333 & 1 & 1 & 0,875 & 0 \\
\hline
\end{tabular}

ISSN (Print): 2456-6411 | ISSN (Online): 2456-6403

\begin{tabular}{|l|l|l|l|l|l|}
\hline & 33 & & & & \\
\hline & 0,956521 & & & 0,928571 & \\
$\mathrm{~S}_{15}$ & 74 & 1 & 1 & 43 & 0 \\
\hline & 0,968873 & 0,924551 & 0,980359 & 0,893599 & 0,075448 \\
AVG & 09 & 93 & 34 & 55 & 07 \\
\hline
\end{tabular}

\subsection{Representation using bar graphs}

Here is our bar chart for the distribution of a quantitative statistical variable. This diagram represents the results of the 15 groups including the average of the observations $[3,7]$ :

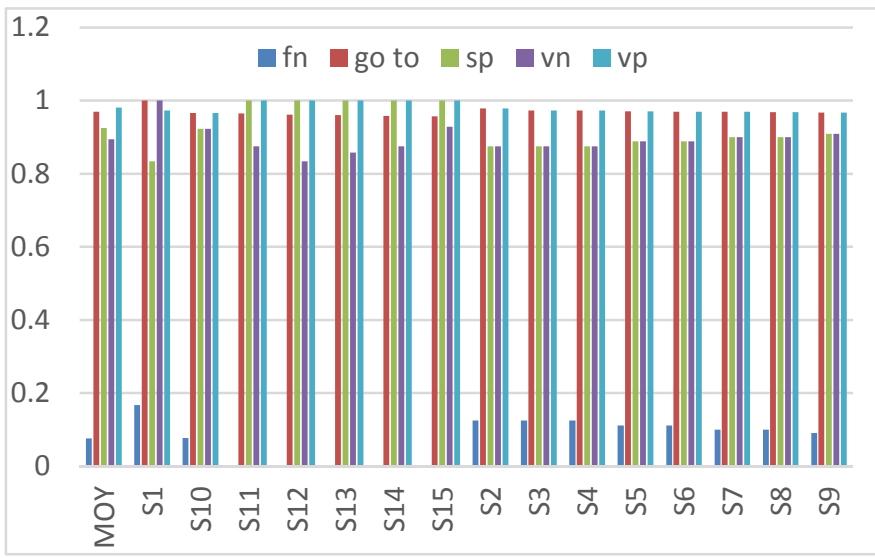

Figure I-2: Bar graph

As we can notice on the figure above, the average values are represented as follows: Sensitivity is 0.96887309 , Specificity is 0.92455193 , Positive predictive value is 0.98035934 , Positive predictive value is 0.89359955 and False Negative $=1-\mathrm{Sp}$ is 0.07544807 .

\subsection{Graphical representation using ROC curve}

The receiver operating characteristic (ROC), also known as the performance characteristic (of a test) or the sensitivity/specificity curve, is a measure of the performance of a binary classifier, i.e. a system whose objective is to categorize items into two distinct groups on the basis of one or more of the characteristics of each of these items [6], [8].

Graphically, the ROC measure is often represented as a curve_that gives the true positive rate (fraction of positives that are actually detected) versus the false positive rate (fraction of negatives that are incorrectly detected).

When a test provides continuous results, the best threshold between the estimated values and the normal values should be determined.

Ideally, sensitivity and specificity should be equal to 1 . This is usually not possible, and the highest values for these two parameters should be sought, knowing that they vary in opposite directions $[6,9]$.

For this choice, we use a graphical tool, the ROC curve (Receiver Operating Characteristics). A ROC curve is a plot of the sensitivity values Se versus 1-Sp. 


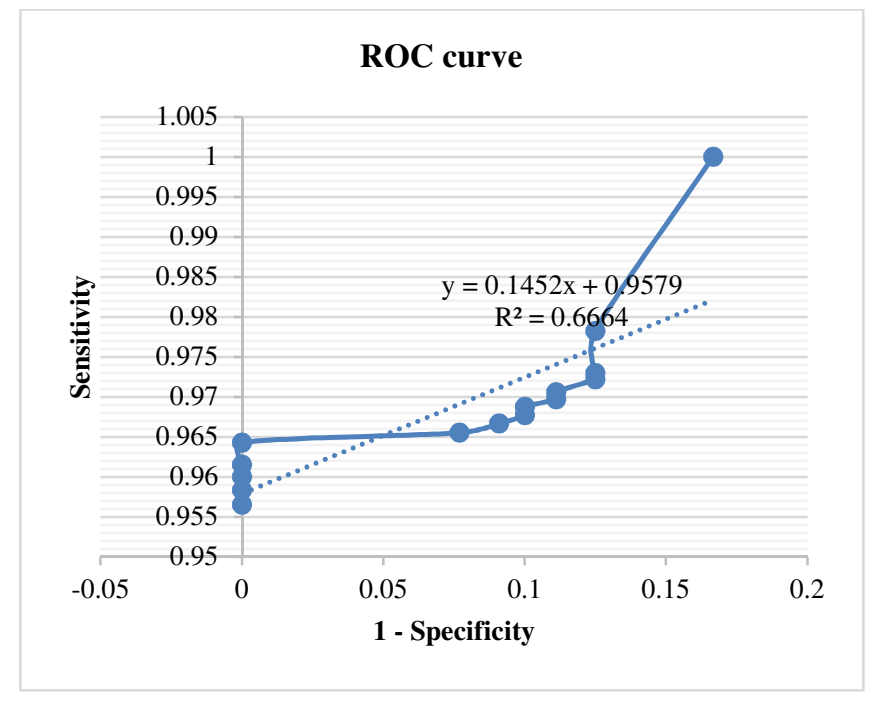

Figure 2-3: ROC curve

According to the performance indicator realized through the ROC curve above, we can confirm that our model is therefore a better classifier that can diagnose whether the footprint is accepted or not with $\mathrm{R}^{2}=0.7$.

\section{CONCLUSION}

Here we are at the end of our work which consisted in evaluating the performance of an access control system to premises secured by fingerprints. To evaluate this performance, we used a regression model in which the dependent variable is of the qualitative type, more precisely, the variable: "the new individual who takes the entry test is positive or negative".

Our model would therefore be a classifier that can diagnose whether the fingerprint is accepted or not. This model for evaluating the performance of the fingerprint-based access control system is carried out by means of the confusion matrix, calculations of the evaluation parameters (Sensitivity, Specificity, positive predictive value, negative predictive value and false negative), and finally plots of the sensitivity values $\mathrm{Se}$ as a function of 1-Sp (ROC curve) [9].

However, we can confirm that our objectives were achieved because on a sample of six hundred individuals of which 470 enrolled and 130 not enrolled, the access control system had the following results: 456 true positives, 14 false negatives, 10 false positives and 120 true negatives which constitutes our confusion matrix, that we were able to evaluate the performance of our system.
From all the tests performed we can conclude that the mean values are represented as follows: Sensitivity is 0.96887309 , Specificity is 0.92455193, Positive Predictive Value is 0.98035934 , Positive Predictive Value is 0.89359955 and False Negative $=1-\mathrm{Sp}$ is 0.07544807 . In terms of success rate of the single mode identification (fingerprint) that we have just performed, we can say that the results obtained are satisfactory since the performance is $96 \%$.

\section{Bibliographic references}

[1]. Houda Benaliouche, Multimodalité Biométrique dans le cadre d'une Application d'Authentification, Thèse de doctorat, Université Ferhat Abbas Sétif 1, Soutenue le : 21 février 2016.

[2]. Ouassima Benouari, Variable selection criterion for mixed conditional logistic regression models when the random effects structure is unknown, LAVAL University, 2019.

[3]. PHILIPPE BESSE, Statistical Learning, Data Science - 3, Institut National des Sciences Appliquées de Toulouse, Toulouse.

[4]. Bopatriciat Boluma Mangata \& al, Contribution of an Embedded and Biometric System in a Replicated Database for Access Control in a MultiEntry Institution, International Journal of Science and Research (IJSR), Volume 10 Issue 3, March 2021.

[5]. Anne-Claire Brunet, Development of statistical tools for the analysis of transcriptomic data by gene co-expression networks, Thesis Presented and defended on June 17, 2016 at THE UNIVERSITY OF TOULOUSE.

[6]. WILLIAM FLAGEOL, PREDICTING DEVELOPMENT AND TESTING EFFORTS FROM USE CASES: AN EXPLORATORY STUDY, University of Quebec at Trois-Rivières, AUGUST 2015.

[7]. Gaurush Hiranandani, Multiclass Performance Metric Elicitation, 33rd Conference on Neural Information Processing Systems (NeurIPS 2019), Vancouver, Canada.

[8]. G. Hiranandani \& al., Performance metric elicitation from pairwise classifier comparisons. In The 22nd International Conference on Artificial Intelligence

and Statistics, pages 371-379, 2019.

[9]. Asma GUIZANI, Treatment of refused files in the process of credit granting to individuals, Doctoral Thesis publicly defended in Paris on: 19 March 2014.

[10]. Frej Limayem, Weighting models by cross sorting methods for collaborative decision support in projects, $\mathrm{PhD}$ thesis presented at ECOLE CENTRALE PARIS, defended on November 23, 2001.

[11]. Ioannis Markoulidakis \& al., Multiclass Confusion Matrix Reduction Method and Its Application on Net Promoter Score Classification Problem, Technologies 2021, 9, 81. https://doi.org/10.3390/technologies9040081.

[12]. H. Narasimhan. Learning with complex loss functions and constraints In International Conference on Artificial Intelligence and Statistics, pages 1646-1654, 2018

[13]. Jasmina Dj. Novakovic \& Al., Evaluation of Classification Models in Machine Learning, Theory and Applications of Mathematics \& Computer Science 7 (1) (2017) 39 - 46.

[14]. Agus Pamuji, Performance of the K-Nearest Neighbors Method on Analysis of Social Media Sentiment, JUISI ISSN: 2460-1306, Vol. 07, No.01, Februari 2021

[15]. Ruuska Salla \& al., Evaluation of the confusion matrix method in the validation of an automated system for measuring feeding behaviour of cattle, Elsevier BV, 2018, http://dx.doi.org/10.1016/j.beproc.2018.01.004.

[16]. Marie-Hélène Simard, Statistical evaluation and forecasting of asset manager performance, LAVAL University, 2015. 